\title{
Broadband Inlet Liner Design for the DGEN Aero-propulsion Research Turbofan
}

\author{
Douglas M. Nark, Michael G. Jones $\dagger^{\dagger}$ and Noah H. Schiller ${ }^{\ddagger}$ \\ NASA Langley Research Center, Hampton, VA 23681-2199, U.S.A \\ Daniel L. Sutliff ${ }^{\S}$ \\ NASA Glenn Research Center, Cleveland, OH 44212, U.S.A
}

\begin{abstract}
The utilization of advanced fan designs (including higher bypass ratios) and shorter engine nacelles has highlighted a need for increased fan noise reduction over a broad frequency range. With these observations in mind, an acoustic liner optimization process has been developed to achieve improved broadband liner designs. A series of advanced liner design studies at increasing technology readiness levels has been conducted as the overall optimization methodology has been enhanced. In this work, further validation was pursued through the fabrication and testing of two liner designs for the NASA Glenn DGEN Aero-propulsion Research Turbofan. Specifically, single and three layer liners were designed, fabricated, and tested. Analysis of the predicted attenuation results showed them to generally match expected trends for the conditions considered. The effects of source distribution on predicted directivity were also illustrated through the flexibility of a statistical source model. As part of the analysis, the overall design and evaluation capability was extended such that external observer locations may be included in the optimization process. This enhancement will provide a much wider design space for the development of advanced broadband liners. These preliminary results provide further confidence in the design tools and enhancements made to the overall liner design process.
\end{abstract}

\section{Nomenclature}

$\begin{array}{ll}c & \text { sound speed } \\ h_{i} & \text { characteristic distance (or height) } \\ i & \sqrt{-1} \\ J_{n} & \text { Bessel function of order } \mathrm{n} \\ k & \text { free-space wave number }(k=\omega / c) \\ m & \text { circumferential mode number } \\ n & \text { radial mode number } \\ p & \text { acoustic pressure normalized by } \rho c^{2} \\ T_{i j} & \text { transmission coefficient } \\ u & \text { acoustic particle velocity normalized by } c \\ W_{i} & \text { weighting values }\end{array}$

\footnotetext{
* Senior Research Scientist, Structural Acoustics Branch, Research Directorate, AIAA Associate Fellow

${ }^{\dagger}$ Senior Research Scientist, Structural Acoustics Branch, Research Directorate, AIAA Associate Fellow

${ }^{\ddagger}$ Research Scientist, Structural Acoustics Branch, Research Directorate

$\S$ Aerospace Engineer, Acoustics Branch, AIAA Associate Fellow
} 
Symbols:

$\begin{array}{ll}\beta & \text { admittance }(\beta=1 / \zeta) \\ \Gamma & \text { propagation constant } \\ \zeta & \text { acoustic impedance normalized by } \rho c(\zeta=\theta+i \chi) \\ \theta & \text { acoustic resistance normalized by } \rho c \\ \mu & \text { mean value } \\ \rho & \text { fluid density } \\ \sigma & \text { square root of the Prandtl number }(\sigma=\sqrt{P r}) \\ \chi & \text { acoustic reactance normalized by } \rho c \\ \Omega & \text { fraction of treated area } \\ \omega & \text { angular frequency }\end{array}$

Abbreviations:

$\begin{array}{ll}\text { AAPL } & \text { Aero-Acoustic Propulsion Laboratory } \\ \text { BPF } & \text { Blade Passage Frequency } \\ \text { CDL } & \text { CDUCT-LaRC } \\ \text { DART } & \text { DGEN Aero-propulsion Research Turbofan } \\ \text { LTF } & \text { Liner Test Facility } \\ \text { MDOF } & \text { Multi-Degree of Freedom } \\ \text { POA } & \text { Percent Open Area } \\ \text { SDOF } & \text { Single-Degree of Freedom } \\ \text { TRL } & \text { Technology Readiness Level } \\ \text { 3DOF } & \text { Three-Degree of Freedom }\end{array}$

\section{Introduction}

The utilization of advanced fan designs (including higher bypass ratios) and shorter engine nacelles has highlighted a need for increased fan noise reduction over a broad frequency range. Thus, improved broadband liner designs must account for these constraints and take advantage of novel liner configurations. With these observations in mind, the development and assessment of a broadband acoustic liner optimization process has been pursued through a series of recent design and experimental studies ${ }^{1 / 6]}$ In this design process, an acoustic duct propagation code is used to predict optimum impedance spectra over a number of frequencies and flow conditions. Acoustic liner modeling tools are then used to identify geometric liner parameters (within manufacturing constraints) necessary to produce impedance spectra that most closely match the predicted optimum values. The resultant impedance values are then used with the propagation code to predict attenuation spectra. Iteration between the prediction and design stages is possible to allow refinement of the liner design (e.g, to account for manufacturing constraints).

To gain further confidence in the tools used within the broadband liner design process, a series of multi-degree of freedom (MDOF) liner design studies at increasing technology readiness levels (TRL) has been conducted as the overall optimization methodology has been enhanced. Initial liner designs targeting the NASA Langley Liner Technology Facility ${ }^{7 / 8}(\mathrm{LTF})$ resulted in predicted optimum impedance spectra consistent with those obtained using higher-fidelity, more computationally intensive models. Subsequent design and testing in the NASA Glenn Advanced Noise Control Fan (ANCF) Rig 3.4 and 9-ft x 15 -ft Low Speed Wind Tunne $\sqrt[516]{6}$ provided further confidence in the design process. Based on these results, further validation was pursued through the fabrication and testing of liner designs for the NASA Glenn DGEN Aero-propulsion Research Turbofan (DART) and is the focus of this work. This latest test configuration and associated measurements are described in Section [I] The computational approach, impedance modeling, and liner design process are then presented in more detail in Sections III, IV, and $\mathrm{V}$, respectively. Evaluation of liner performance and comparison with predictions are provided in Section VI Finally, concluding remarks regarding some of the more significant results and further areas of interest are presented in Section/VII 


\section{Test Facility and Acoustic Measurements}

Recently, the DART has become operational in the NASA Glenn Aero-Acoustic Propulsion Laboratory (AAPL). This afforded the opportunity to further validate the liner design process and assess recent MDOF configurations under consideration for future flight testing. The DART is based on the Price Induction DGEN380, which is a small (length of 44.3 in $(1.13 \mathrm{~m})$ and fan inlet diameter of 13.78 in $(.35 \mathrm{~m})$ ) approximately 500-lbf thrust class, geared turbofan engine with a separate flow nozzle and a bypass ratio of 7.6. In order to increase the amount of surface area available for acoustic treatment, an inlet spool was designed and manufactured by Price Induction to accommodate acoustic liners. As shown in Figure 2, this spool had a pocket extending the outer flow path and incorporating a removable end cap to facilitate liner insertion/removal. A hardwall extension of the same axial dimension was also manufactured to provide consistent comparison of hardwall and treated results. Measurements were made on a 10-ft. arc array as shown in Figure 3 . Further discussion of the DART and AAPL may be found in a paper by Brown and Sutliff. 9

\section{Acoustic Predictions}

For the current investigation, the majority of the duct propagation and radiation predictions were conducted using the CDUCT-LaRC (CDL) code. This code calculates the propagation of a given acoustic source ahead of the fan face or aft of the exhaust guide vanes in the inlet or exhaust ducts, respectively. Subsequent to the propagation calculations, the code has the capability of computing the noise radiation field outside the duct. The three-dimensional duct may include acoustic treatment (possibly circumferentially and radially segmented) and incorporate struts/bifurcations. All of the modules that currently make up the CDL framework have been discussed previously $\sqrt{10}$ and are utilized in this study. However, this discussion will focus on the propagation and radiation modules, as they are most pertinent to this investigation.

The duct propagation module is based on the CDUCT code developed by Dougherty ${ }^{11} \mid 12$ and extended by Lan. ${ }^{13}$ This code utilizes a parabolic approximation to the convected Helmholtz equation and offers a computationally efficient model that accounts for the complexities of fully three-dimensional nacelle configurations. To provide for such prediction capability, the CDL code supports multi-block propagation calculations in which grid connectivity is determined and data is transferred from upstream to downstream blocks without user intervention. ${ }^{10}$ Results of the propagation module include the acoustic potential or pressure within the duct, which may be utilized by the radiation module for acoustic radiation calculations.

The duct radiation model is based on the Ffowcs Williams-Hawkings (FW-H) equation with a permeable data surface ${ }^{14}$ Based on the background flow conditions and propagation solution, this module calculates the radiated acoustic pressure at various observer locations. Currently, the data surface is taken to be the nacelle inlet or exhaust plane for inlet or aft-fan cases, respectively. However, provisions have also been made to attempt to account for the effects of the shear layer on the radiation.

While CDL can accept arbitrary source specification (i.e., it is not a modal code), it is convenient to specify the acoustic source distribution in terms of duct modes. For situations in which the source pressure is available, this greatly simplifies conversion to the required acoustic potential. However, when source information is not available, an assumption on the source description must be made. This is generally the case and the approach taken here follows that described by Zlavog and Eversman 15,16 in a series of statistical studies into the effects of randomized modal source power and/or phase on attenuation in lined ducts. In this study, the source modal powers (and hence, amplitudes) and modal phases are allowed to vary randomly and independently. Thus, with equal probability of occurrence, the amplitude and phase for each cut-on source mode may take any value in the intervals from 0 to 1 and 0 to $2 \pi$, respectively. This uncertainty in source specification will undoubtedly result in uncertainty in the predicted sound levels. However, as discussed by Zlavog and Eversman, $\frac{15,16}{1}$ transmitted power appears to be normally distributed if the source includes all propagating circumferential and radial acoustic modes with random modal power and phase. In their studies, such a source specification also produced statistical distributions of transmitted power with the smallest standard deviation. Therefore, as discussed in a previous CDL study ${ }^{17}$ Student's t-distribution is used to statistically analyze the predicted attenuation results. For each configuration and condition, 11 simulations (with randomized amplitude and phase for all cut-on source modes) were performed to produce a sample population from which statistical information can be inferred.

As with previous liner optimization studies $\frac{1135}{\sqrt{135}}$ the cost function was formulated to maximize in-duct attenuation. Thus, optimum impedance values were predicted using the propagation module of the CDL code (with the aforementioned source model) coupled with optimization routines. This was accomplished by using the Python programming language (http://www.python.org) to "wrapper" the CDL calculations. The use of the Python scripts allows access to 
the full set of tools available in the optimization package of the Scipy ${ }^{18}$ library.

As part of a continuing effort to add to the prediction tools available to the Langley Liner Physics Team, the COMSOL code ${ }^{19}$, 20 was used to confirm selected in-duct CDL propagation predictions. Similar to CDL, COMSOL solves the convected Helmholtz equation with the acoustic potential as the unknown variable. However, a conventional Galerkin finite element method is used and the effects of reflections at the leading and trailing edges of the liner are captured. In addition, there is the potential to capture reflections at the duct termination. However, in this study, a 2D axisymmetric model was used with a perfectly matched layer boundary condition to approximate an anechoic termination.

\section{Impedance Prediction Model}

The impedance prediction model used in this study combines two models presented in an earlier paper. ${ }^{21}$ The first is a transmission line mode ${ }^{22}$ that assumes acoustic wave propagation through each layer of the liner (see Fig. 44, and the second is a lumped element mode $e^{23-25}$ used to compute the impedance change across perforates. The normalized surface impedance spectra presented by each chamber of the liner are computed separately, and are then combined to determine an effective surface impedance spectrum that is assumed uniform across the liner surface.

\section{A. Single Chamber Computations}

The transmission line model for predicting the surface acoustic impedance of an individual liner chamber is briefly described herein with the aid of Fig. 4. This figure provides a sketch of a single chamber of a liner with two air gaps (sections with heights of $h_{1}$ and $h_{3}$ ) and two perforate sheets (thicknesses of $h_{2}$ and $h_{4}$ ). The thickness of each perforate sheet is exaggerated to demonstrate distinct features of the models used in this study. This model treats each unique portion of the liner chamber separately via a transmission line computation. In the following discussion, these unique portions are treated as individual "computational layers."

The transmission line computation is initiated by assuming the backplate at $x_{0}$ to be rigid and impermeable. The normalized acoustic pressure and particle velocity are given as

$$
\left(\begin{array}{l}
p_{0} \\
u_{0}
\end{array}\right)=\left(\begin{array}{l}
1 \\
0
\end{array}\right)
$$

which yields an infinite impedance at the backplate of the liner. All acoustic pressures and particle velocities are normalized by $\rho c^{2}$ and $c$, respectively, where the density of the air and the speed of sound are for the prevailing static pressure and air temperature. Correspondingly, impedances are normalized by the characteristic impedance of air, $\rho c$. Changes in the acoustic pressure and particle velocity across a computational layer are computed via

$$
\left(\begin{array}{l}
p_{n+1} \\
u_{n+1}
\end{array}\right)=\left(\begin{array}{ll}
T_{11} & T_{12} \\
T_{21} & T_{22}
\end{array}\right)\left(\begin{array}{l}
p_{n} \\
u_{n}
\end{array}\right)
$$

where the transmission coefficients $\left(T_{11}, T_{12}, T_{21}\right.$ and $\left.T_{22}\right)$ depend on the modeling approach and the type of layer being considered.

For open layers (air gaps), the transmission coefficients are given by

$$
T_{11}=T_{22}=\cosh (k \Gamma h) ; T_{12}=\zeta_{c} \sinh (k \Gamma h) ; T_{21}=\zeta_{c}^{-1} \sinh (k \Gamma h)
$$

where $k=\omega / c$ is the free-space wavenumber, $\omega$ is the angular frequency, and $h$ is the layer thickness. The propagation constant, $\Gamma$, and characteristic impedance, $\zeta_{c}$, are computed as

$$
\Gamma=\sqrt{\frac{J_{0}\left(i^{3 / 2} s\right)}{J_{2}\left(i^{3 / 2} s\right)}} \sqrt{\frac{\gamma}{n_{\Gamma}}} ; \quad \zeta_{c}=\frac{-i}{\Gamma} \frac{J_{0}\left(i^{3 / 2} s\right)}{J_{2}\left(i^{3 / 2} s\right)}
$$

where

$$
n_{\Gamma}=\left[1+\frac{\gamma-1}{\gamma} \frac{J_{0}\left(i^{3 / 2} \sigma s\right)}{J_{0}\left(i^{3 / 2} \sigma s\right)}\right]^{-1}
$$


$i=\sqrt{-1}$ is the imaginary unit, $s=\left(d_{c} / 2\right) \sqrt{\rho_{s} \omega / \mu}$ is the shear wave number, and $d_{c}, \rho_{s}, \omega, \mu, \sigma$ and $\gamma$ are the channel diameter, static density, angular frequency, coefficient of viscosity, square root of the Prandtl number and specific heat ratio, respectively. Note that if a liner chamber only contains a single open computational layer (cavity with no facesheet), the normalized surface impedance for that chamber is computed as

$$
\zeta_{c h}=\frac{p_{1}}{u_{1}}
$$

For those cases where the liner chamber contains more than one computational layer, the acoustic pressure and particle velocity computed at the top of the first computational layer $\left(p_{1}\right.$ and $\left.u_{1}\right)$ are input back into Eq. 2 to compute the impedance change across the next computational layer.

Two approaches are considered for computing the change in impedance across an embedded septum, assumed to consist of either a perforated sheet or a wire mesh. The first assumes the septum to be a purely resistive lumped element that is too thin to support wave propagation. For this approach, the transmission coefficients for Eq. 2 are given by

$$
T_{11}=T_{22}=1 ; \quad T_{12}=\frac{R_{f}}{\rho c} ; \quad T_{21}=0
$$

where the normalized DC flow resistance, $R_{f} / \rho c$, across the perforated sheet is often estimated from a lumped element, semi-empirical model (e.g., the Two-Parameter Impedance Prediction Model ${ }^{24}$ ).

The second approach assumes the perforated sheet to be sufficiently thick to support wave propagation. Figure 4 is particularly useful to describe this approach. It is assumed that Eqs. 2 and 3 have been used to determine the acoustic pressure and particle velocity ( $p_{1 a}$ and $u_{1 a}$ ) at the top of the lower air gap. The acoustic pressure and acoustic mass flow are assumed to be constant across the $\{1 a, 1 b\}$ interface, such that

$$
p_{1 b}=p_{1 a} ; \quad N S_{1 b} u_{1 b}=S_{1 a} u_{1 a}
$$

where $N$ is the number of orifices ( 3 in this example) connected to a single air cavity, $S_{1 a}$ is the cross-sectional area at the top of the air cavity (denoted by the long dashed line at the top of the cavity) and $S_{1 b}$ is the cross-sectional area within a single perforate orifice. The wave propagation within the single orifice is then computed using Eqs. 2 and 3 . where the orifice diameter is used as the "channel diameter." Again, the acoustic pressure and acoustic mass flow are assumed to be constant across the $\{2 a, 2 b\}$ interface, such that

$$
p_{2 b}=p_{2 a} ; \quad S_{2 b} u_{2 b}=N S_{2 a} u_{2 a}
$$

Regardless of which approach is used to model embedded septa (for those configurations that contain them), the process described above is repeated until the acoustic pressure and particle velocity are determined at $x_{3}$ (the plane just below the perforated facesheet). The normalized impedance at $x_{3}$ is then given by

$$
\zeta_{n-1}=\frac{p_{n-1}}{u_{n-1}}
$$

( $n=4$ for the example given in Fig. 4). The Two-Parameter Impedance Prediction Model is then used to predict the impedance change across the perforated facesheet, such that the effects of grazing flow over the surface of the liner can be included. The resultant normalized surface impedance for the chamber (at the plane denoted by the dashed line with endpoints $A$ and $B$ ) is given by

$$
\zeta_{c h}=\frac{p_{n}}{u_{n}}
$$

where $n$ represents the number of computational layers in the chamber.

\section{B. Liner Surface Impedance Computation}

The surface impedance spectra of the individual chambers are combined to compute the effective impedance across the liner surface. For this computation, it is preferable to use acoustic admittance $\left(\beta_{c h}=1 / \zeta_{c h}\right)$. The effective admittance across a selected extent of liner surface is given by

$$
\beta_{s}=\Omega \sum_{i=1}^{N_{c h}} \beta_{c h}
$$


where $N_{c h}$ represents the number of chambers that combine to form the liner and $\Omega$ is the fraction of the surface that is comprised of this group of chambers. The uniform, effective, surface impedance of the liner is then given by $\zeta_{s}=1 / \beta_{s}$.

\section{Liner Design}

As indicated above, the CDL propagation code was used to determine target optimum impedance values, $\zeta_{\text {opt }}\left(f_{i}\right)$, at selected frequencies, $f_{i}$, for a given flow speed over a design space encompassing $0<\theta \leq 5$ and $-10 \leq \chi \leq 10$. With this being the first operational test of DART, limited information was available on DART source content and flow conditions. Therefore, target values were obtained at one-third octave center frequencies ranging from $1000 \mathrm{~Hz}$ to $8000 \mathrm{~Hz}$ at a flow condition expected to be nominally representative of $100 \%$ of design RPMc $(M=0.5$ at the fan face). The efficiency of this process allows for parallel-element, variable-impedance, broadband liner concepts at various technology readiness levels (TRL) to be considered for implementation. Therefore, it is expected that updated liner designs with improved broadband noise reduction will be produced as additional DART information becomes available.

To achieve the desired broadband performance, candidate liner designs consisted of multi-layer configurations incorporating septa (or "mesh-caps") embedded into a honeycomb core. ${ }^{21}$ This concept allows the acoustic liner to be customized such that the surface impedance of each individual cell is independently controlled. This is achieved by the combination of parameters used to set the impedance in each cell. Clearly, manufacturing constraints (e.g., liner geometric parameters such as porosity and core depth) are key ingredients in this modeling phase, and must be taken into account to design acoustic liners that can be realistically achieved. In this study, the honeycomb cells were allowed to contain up to two mesh-caps. The mesh cap depths, as well as DC flow resistances, were allowed to vary from cell to cell. The liner modeling tools were used to obtain design impedance values, $\zeta_{\text {pred }}\left(f_{i}\right)$, that minimize the function

$$
F=\left\{\sum_{i=1}^{N} W_{i}\left(\zeta_{\text {opt }}\left(f_{i}\right)-\zeta_{\text {pred }}\left(f_{i}\right)\right)\left(\zeta_{\text {opt }}\left(f_{i}\right)-\zeta_{\text {pred }}\left(f_{i}\right)\right)^{*}\right\}^{0.5} .
$$

Here, the values $W_{i}$ are weighting factors that may be used to assign increased significance to a selected number of frequencies and/or flow conditions and $N$ is the total number of frequency/flow condition combinations considered (in this case, $N=10$, as only one flow condition was considered). Note that with $W_{i}=1$ for all $N$, equation 13 reduces to the $L_{2}$ norm of the difference between the predicted optimum and the design impedance values. As with the first step of the design process, the liner modeling tools were also coupled with the Scipy ${ }^{18}$ optimization routines. This replaced the "brute-force" optimization process used previously for this stage of the design process and provided increased efficiency, as well as access to a much wider design space in matching the target impedance spectra.

Based on the above cost function, a number of MDOF liner concepts were considered. However, manufacturing liners for the DART inlet represents a unique challenge. Because of the small engine diameter ( 14 inches), it becomes a significant challenge to bend the core to achieve a flush fit. This limited some of the choices for manufacturing of the liners. Therefore, consideration was made to select liners allowing acquisition of sufficient data to demonstrate the validity of the liner design process, and to gain insight that could be used to better optimize liners for future tests with this engine. Ultimately, two liner concepts with total core depths of $1.936 \mathrm{in}(49.2 \mathrm{~mm})$ and $0.032 \mathrm{in}(0.8 \mathrm{~mm})$ thick 8\% POA facesheets were selected for this study. The first, shown in Figure 5a is a 2DOF liner that contains a septum located 0.23 in $(5.8 \mathrm{~mm}$ ) from the backplate with a resistance (2000 MKS Rayls) assumed sufficiently high to effectively eliminate sound transmission into the lower chamber. Thus, from an acoustics point of view, it should be considered as an SDOF liner. The second, shown in Figure 5b, is a 3DOF liner that contains two septa, located 0.723 in $(18.4 \mathrm{~mm})$ and 1.716 in $(43.6 \mathrm{~mm})$ from the backplate, in each honeycomb chamber. Each septa has the same resistance (500 MKS Rayls) and the same distribution is used in each chamber.

A comparison of the predicted mean in-duct attenuation at the design flow condition $(M=0.5)$ for both liners is presented in Figure 6 As might be expected, the 3DOF design is predicted to outperform the SDOF design over almost the full frequency range. However, it can be seen that separation between the prediction curves is diminished in the upper frequency range, illustrating the challenge of obtaining larger attenuation in the DART inlet. In addition to the statistical source results, CDL and COMSOL in-duct attenuation predictions for the 3DOF liner using individual source modes are presented in Figure 7. As a starting point for comparison, these cases are based on the specification of a single circumferential and radial ( $m, n$, respectively) source mode. As seen in the figure, the results compare nicely and provide some confidence in the in-duct predictions used for design purposes. 


\section{Results and Discussion}

The two liners described above were fabricated and tested on the DART in the AAPL facility ${ }^{9}$ and an example of the initial test results is provided in Figure 8 Here, the total Power Level (PWL) insertion loss for each liner at observers on the 10-ft. arc array (see Figure 3 ) is presented over a range of fan speeds (RPMc). Overall, the liner designs appear to perform as expected. However, as initial results at the various fan speeds were further analyzed, it became evident that the mean flow Mach number $(M=0.5)$ used in the design process was too high. At the highest corrected fan speed tested (92.5\% RPMc due to ambient conditions and the addition of an internal containment shield for safety requirements in the research environment), flow measurements indicate the mean flow Mach number to be more in the range $M=0.35$. Therefore, the predicted impedances (based on the models in Section IV] for the two liners needed to be adjusted before direct comparisons could be made at any fan speed. In addition, the Mach number disparity also means that the test points all represent off-design conditions. It should be noted that this does not negate the usefulness of the testing. As mentioned previously, consideration was made to select liners allowing acquisition of sufficient data to demonstrate the validity of the liner design process, and to gain insight that could be used to better optimize liners for future tests with this engine. Therefore, while maximum attenuation may not be achieved, a great deal of information remains to be gained through comparison of predicted and measured results.

In order to perform such comparisons on the 10-ft. arc array, the prediction process used in the liner design must be extended. Recall that in-duct attenuation was chosen as the cost function for the impedance optimization. As described in Section III], these in-duct propagation predictions are radiated to arc observer locations using the CDL FW-H duct radiation model. Here, the nacelle inlet plane (exit plane for inlet propagation) is used as a permeable data surface. In keeping with the statistical source model, radiation calculations are performed for each source distribution (random amplitude and phase). Thus, 11 radiation simulations are performed over the full arc array to produce a sample population from which statistical information can be inferred. An example result for the hardwall, SDOF, and 3DOF inlet configurations at $f=3150 \mathrm{~Hz}$ (one-third octave band center frequency) and 90\% RPMc are presented in Figure 9 . In this figure, the directivity patterns are displayed as "normalized" SPL levels (the difference between the SPL and the hardwall peak SPL since relative levels are of interest. In addition, errors bars denoting a range of +/- one standard deviation are included on the hardwall results to illustrate the statistical nature of the result. Similar standard deviation values were obtained for the treated configurations, but are not included to clarify the presentation. Note that the same set of modal source distribution (same randomization seed) was used for each configuration. Generally, the attenuation trends in this example appear to follow those in the in-duct prediction with the 3DOF providing improved performance over the full directivity range.

To further illustrate the flexibility of the statistical source approach, predicted mean attenuations (hardwall SPL treated SPL) for the SDOF and 3DOF liner are presented in Figure 10 at $f=3150 \mathrm{~Hz}, 90 \%$ RPMc for two different source representations (with and without the plane wave included). Again, the generally improved performance of the 3DOF is evident. In addition, the effect of including the plane wave in the source specification can be seen in the resulting change in attenuation over various observer angles. A similar result is seen in Figure 11 at $f=6300 \mathrm{~Hz}$ (one-third octave band center frequency) and 90\% RPMc. Thus, with this capability in place, future work will entail the use of this prediction process over the full fan speed and frequency range for comparison with measured results. Additionally, the effects of modal source distribution will be investigated.

\section{Concluding Remarks}

This paper describes extension of a broadband acoustic liner optimization design process to the DART installed in AAPL. Based on in-duct attenuation predictions, SDOF and 3DOF liners were designed, fabricated, and tested. During the testing, it was discovered that the RPMc had to be constrained such that the mean flow in the DART did not achieve the liner design point Mach number. Consequently, it is recognized that the liner design is not fully optimized for the actual test conditions. Nevertheless, a great deal of information can be gained through comparison of predicted and measured results. A cursory analysis of predicted attenuation results showed them to generally match expected trends (with the 3DOF liner providing improved performance) for the conditions considered. The effects of source distribution on predicted directivity were also illustrated through the flexibility of the statistical source model.

As part of the comparison process, the overall design and evaluation capability was extended such that external observer locations may be included in the optimization process. This enhancement will provide a much wider design space in designing advanced broadband liners. These preliminary results provide further confidence in the design tools, as well as the enhancements made to the overall liner design process. Future work will entail the use of this prediction process over the full fan speed and frequency range for comparison with measured results on the 10-ft. arc 
array. This will provide an excellent opportunity to further evaluate the broadband liner design tools.

\section{Acknowledgments}

This research was funded by the Advanced Air Transport Technology (AATT) Project of the NASA Advanced Air Vehicles Program (AAVP).

\section{References}

${ }^{1}$ Nark, D. M. and Jones, M. G., "Broadband Liner Optimization for the Source Diagnostic Test Fan,” AIAA Paper 2012-2195, 2012.

${ }^{2}$ Nark, D. M., Jones, M. G., Sutliff, D. L., Ayle, E., and Ichihashi, F., "Improved Broadband Liner Optimization Applied to the Advanced Noise Control Fan," AIAA Paper 2014-3103, 2014.

${ }^{3}$ Nark, D. M., Jones, M. G., and Sutliff, D. L., "Modeling of Broadband Liners Applied to the Advanced Noise Control Fan," AIAA Paper 2015-2693, 2015.

${ }^{4}$ Sutliff, D. L., Jones, M. G., and Nark, D. M., "In-Duct and Far-field Experimental Measurements from the ANCF for the Purpose of Improved Broadband Liner Optimization,” AIAA Paper 2014-3231, 2014

${ }^{5}$ Nark, D. M., Jones, M. G., and Sutliff, D. L., "Further Development and Assessment of a Broadband Liner Optimization Process," AIAA Paper 2016-2784, 2016.

${ }^{6}$ Sutliff, D. L., Nark, D. M., and Jones, M. G., "Efficacy of a Multiple Degree of Freedom Acoustic Liner Installed in the Bypass of a Scale Model High Speed Fan," AIAA Paper 2016-3005, 2016.

${ }^{7}$ Gerhold, C. H., Brown, M. C., and Jones, M. G., "Segmented Liner to Control Mode Scattering," AIAA Paper 2013-2078, 2013.

${ }^{8}$ Jones, M. G., Watson, W. R., and June, J. C., "Optimization of Microphone Locations for Acoustic Liner Impedance Eduction," AIAA Paper 2015-3271, 2015.

${ }^{9}$ Brown, C. and Sutliff, D. L., "DGEN Aeropropulsion Research Turbofan (DART): Projecting Noise to the Far-Field and Baseline Acoustics," Tech. Rep. 2018-xxxx, AIAA, 2018.

${ }^{10}$ Nark, D. M., Farassat, F., Pope, D. S., and Vatsa, V., "The Development of the Ducted Fan Noise Propagation and Radiation Code CDUCTLaRC," AIAA Paper 2003-3242, 2003.

${ }^{11}$ Dougherty, R. P., “A Wave-Splitting Technique for Nacelle Acoustic Propagation,” AIAA Paper 97-1652, 1997.

${ }^{12}$ Dougherty, R. P., "A Parabolic Approximation for Flow Effects on Sound Propagation in Nonuniform, Softwall, Ducts," AIAA Paper 99$1822,1999$.

${ }^{13}$ Lan, J. H., “Turbofan Duct Propagation Model,” NASA CR 2001-211245, 2001.

${ }^{14}$ Brentner, K. B. and Farassat, F., "Analytical Comparison of the Acoustic Analogy and Kirchhoff Formulation for Moving Surfaces," AIAA Journal, Vol. 36, No. 8, 1998, pp. 1379-1386.

${ }^{15}$ Zlavog, G. and Eversman, W., "Source effects on attenuation in lined ducts. Part I: A statistically based computational approach," Journal of Sound and Vibration, Vol. 307, No. 1-2, 2007, pp. 113-138.

${ }^{16}$ Zlavog, G. and Eversman, W., "Source effects on attenuation in lined ducts. Part II: Statistical properties," Journal of Sound and Vibration, Vol. 307, No. 1-2, 2007, pp. 139-151.

${ }^{17}$ Nark, D. M., "Assessment of Radiated Fan Noise Prediction Capabilities using Static Engine Test Data," AIAA Paper 2011-2807, 2011.

${ }^{18}$ Jones, E., Oliphant, T., Peterson, P., et al., "SciPy: Open source scientific tools for Python," 2001 -.

${ }^{19}$ Jones, M. G., Watson, W. R., Nark, D. M., and Schiller, N. H., "Evaluation of Spanwise Variable Impedance Liners with Three-Dimensional Aeroacoustics Propagation Codes," AIAA Paper 2017-3021, AIAA, 2017.

${ }^{20}$ COMSOL Acoustic Module User's Guide, v5.3a ed., 2018.

${ }^{21}$ Jones, M. G., Howerton, B. M., and Ayle, E., "Evaluation of Parallel-Element, Variable-Impedance, Broadband Acoustic Liner Concepts," AIAA Paper 2012-2194, 2012.

${ }^{22}$ Parrott, T. L. and Jones, M. G., "Parallel-Element Liner Impedances for Improved Absorption of Broadband Sound in Ducts," Noise Control Engineering Journal, Vol. 43, No. 6, 1995, pp. 183-195.

${ }^{23}$ Motsinger, R. E. and Kraft, R. E., "Design and Performance of Duct Acoustic Treatment: Aeroacoustics of Flight Vehicles; Chapter 14, Vol. 2: Noise Control," NASA RP 1258, August 1991.

${ }^{24}$ Jones, M. G., Parrott, T. L., and Watson, W. R., "Uncertainty and Sensitivity Analyses of a Two-Parameter Impedance Prediction Model," AIAA Paper 2008-2928, 2008.

${ }^{25}$ Parrott, T. L. and Jones, M. G., "Assessment of NASA's Aircraft Noise Prediction Capability, Chapter 6: Uncertainty in Acoustic Liner Impedance Measurement and Prediction," NASA TP 2102-215653, July 2012. 


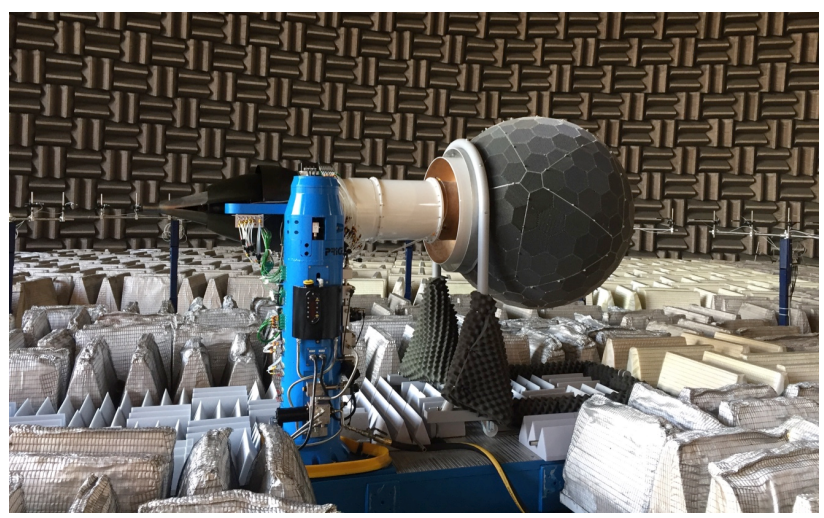

(a) DART with hardwall inlet extension installed.

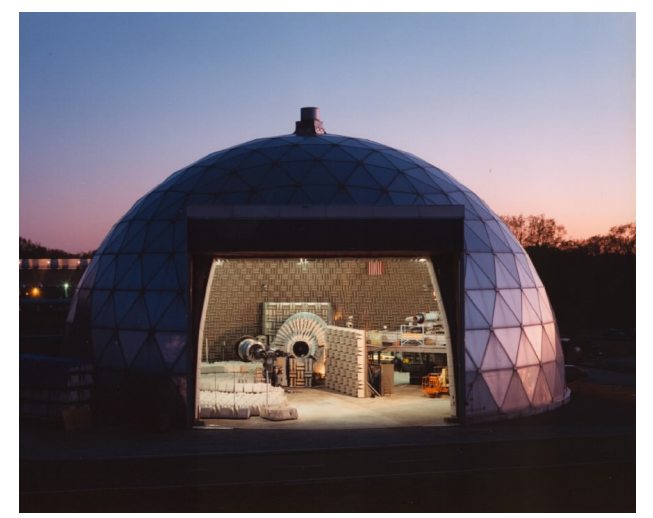

(b) AAPL.

Figure 1: Photos of the DART and AAPL facility.

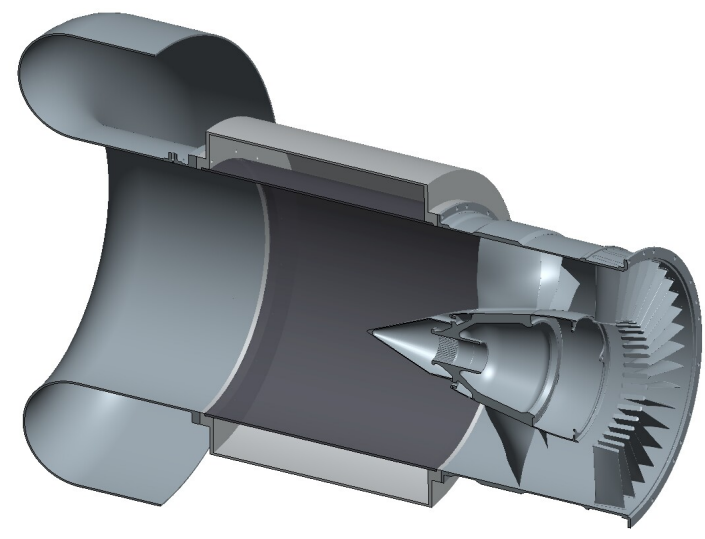

Figure 2: DART inlet liner assembly.

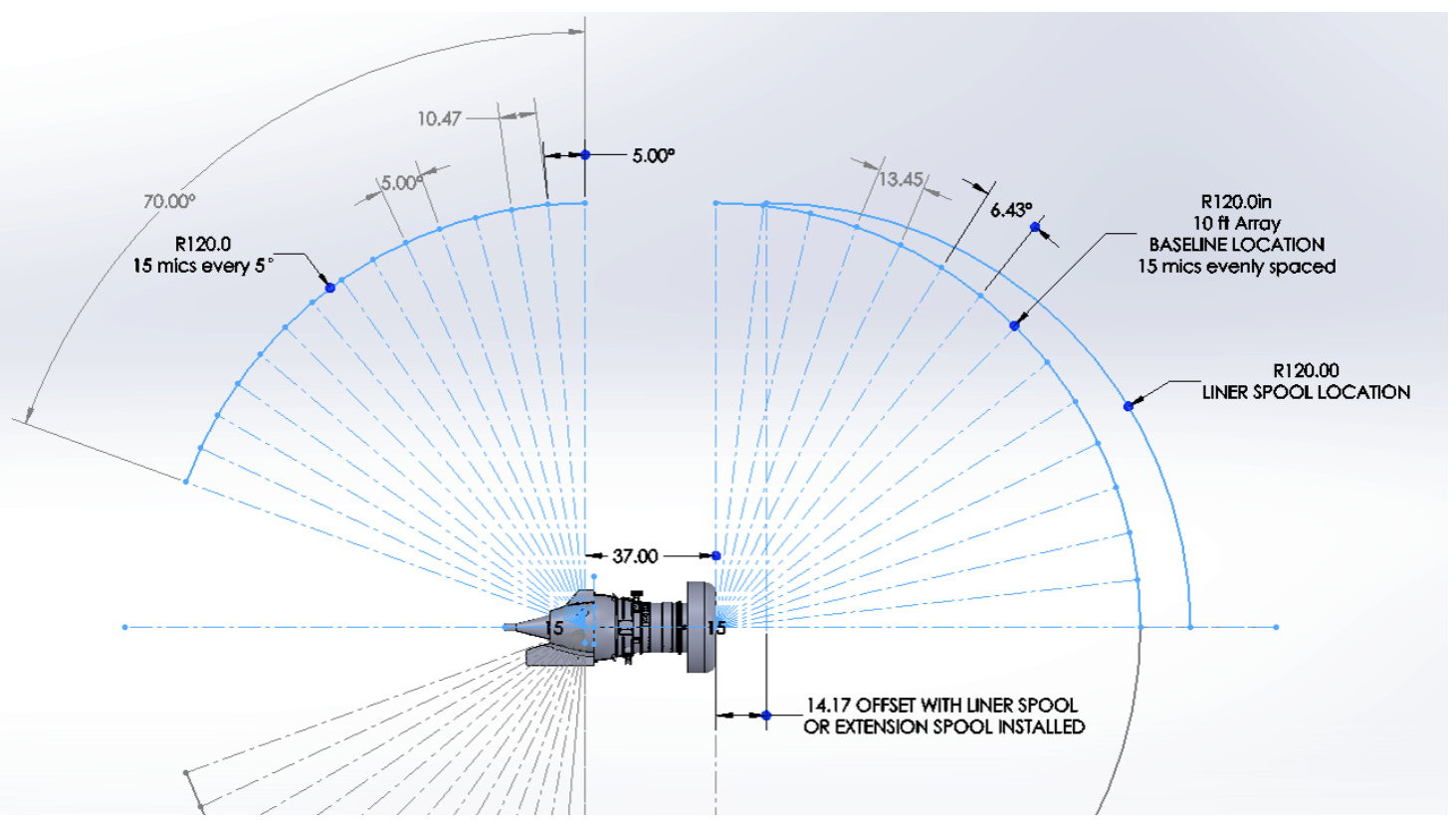

Figure 3: Arc array positioned for DART measurements. 


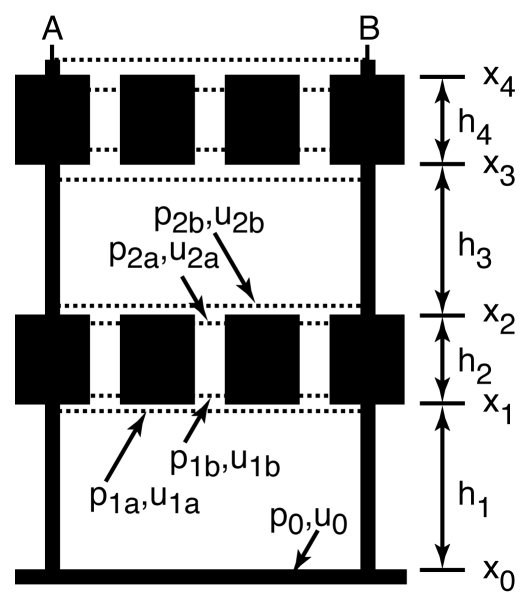

Figure 4: Sketch of single chamber of a liner with key parameters for transmission line impedance prediction model.

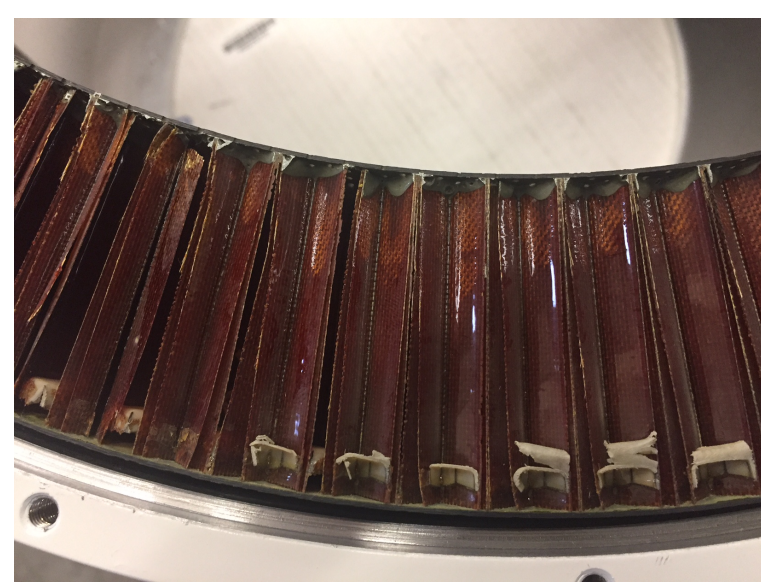

(a) Nominal SDOF liner (very high resistance septa).

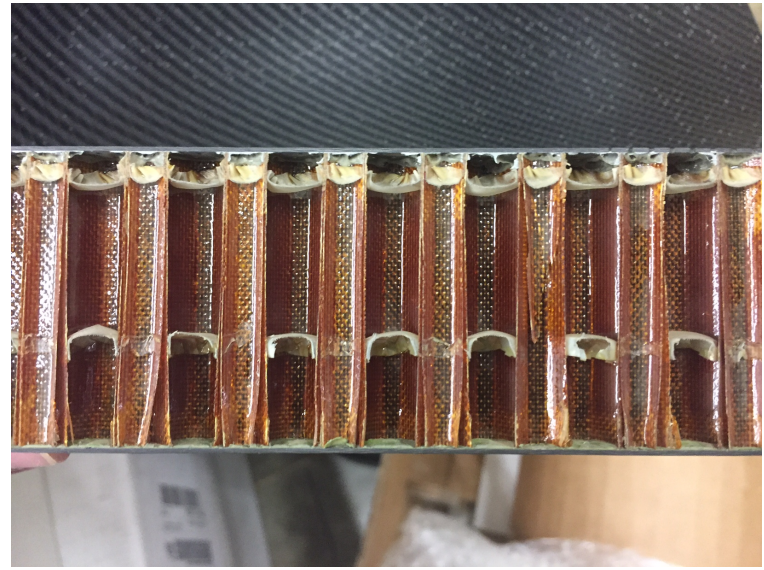

(b) 3DOF liner.

Figure 5: Acoustic liner samples.

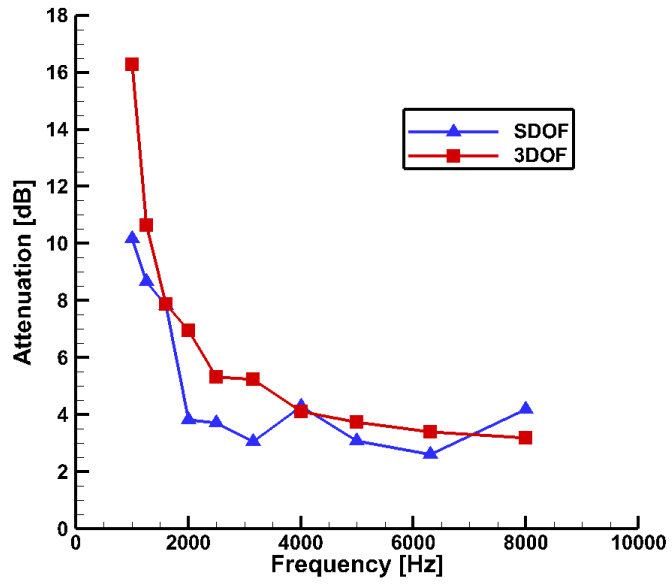

Figure 6: Predicted mean attenuation for the SDOF and 3DOF liners at the design conditions $(M=0.5)$. 


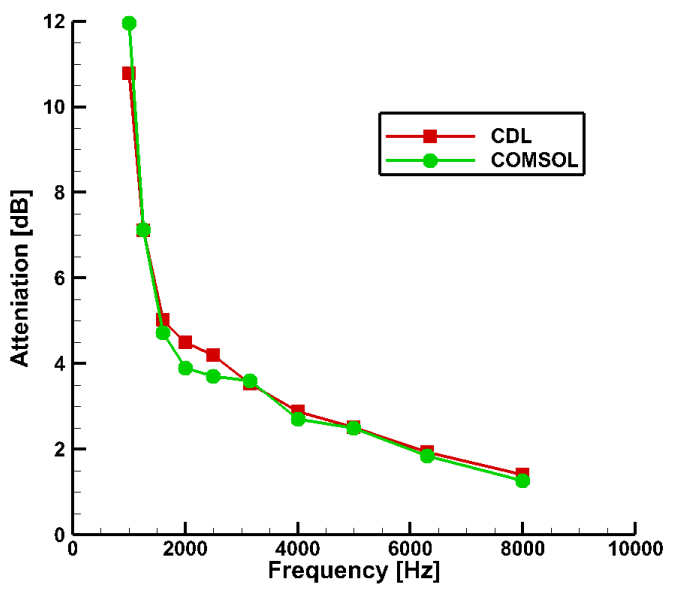

(a) Source Mode: $m, n=0,0$ (plane wave).

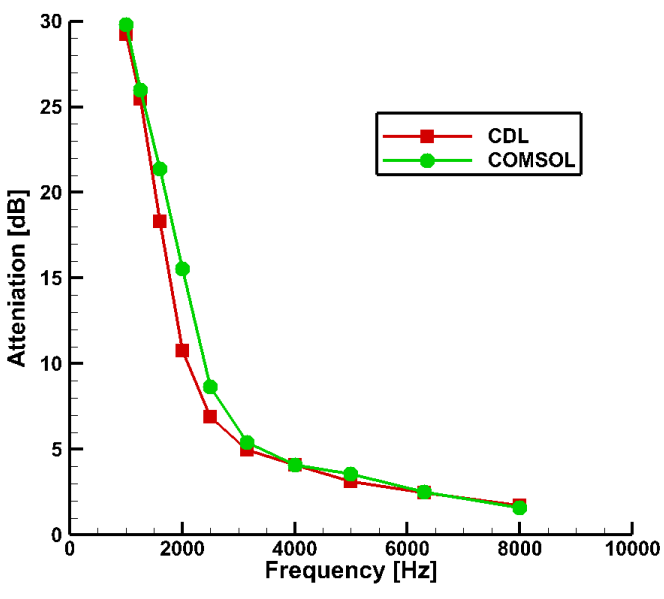

(b) Source Mode: $m, n=2,1$.

Figure 7: Comparison of CDL and COMSOL in-duct attenuation predictions for the 3DOF design using individual source modes.

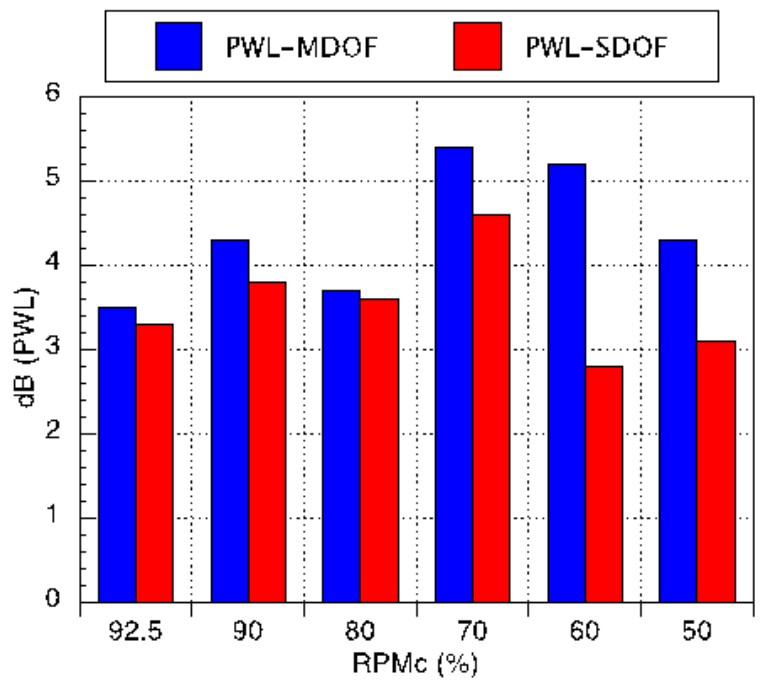

Figure 8: Measured Total PWL insertion loss. 


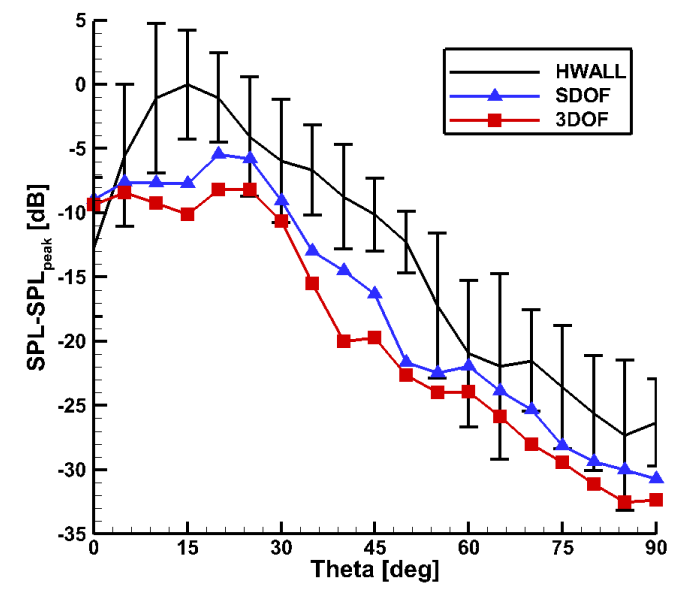

Figure 9: Predicted mean directivity on the 10-ft. arc array at $f=3150 \mathrm{~Hz}(90 \% \mathrm{RPMc})$ for hardwall and treated inlets. Source specification includes all cut-on modes with random amplitude and phase (excluding the plane wave) and error bars on hardwall results denote a range of \pm one standard deviation.

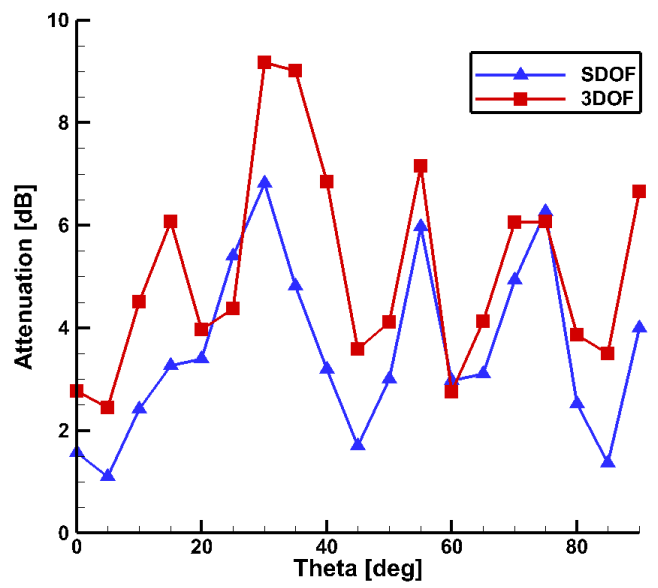

(a) Plane wave included in source specification.

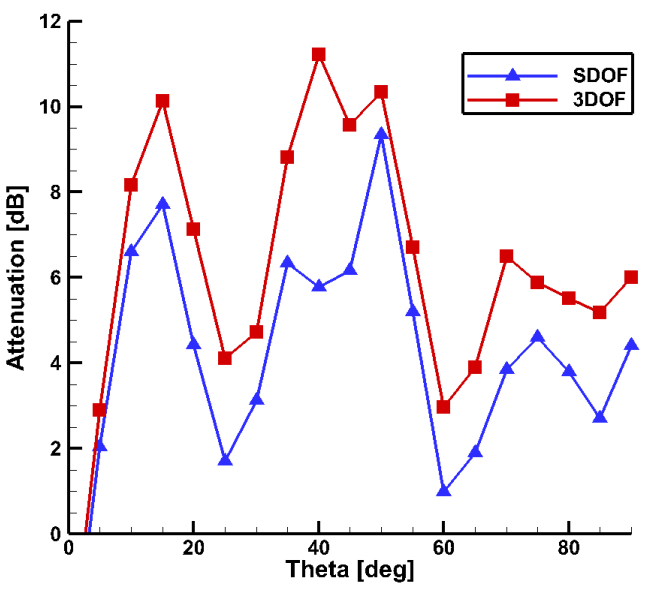

(b) Plane wave neglected in source specification.

Figure 10: Predicted mean CDL attenuation on the 10-ft. arc array for treated inlets at $f=3150 \mathrm{~Hz}(90 \% \mathrm{RPMc})$. 


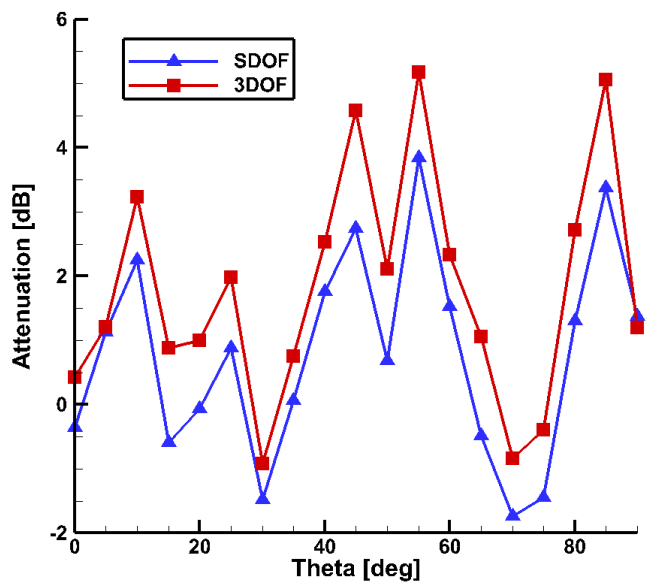

(a) Plane wave included in source specification.

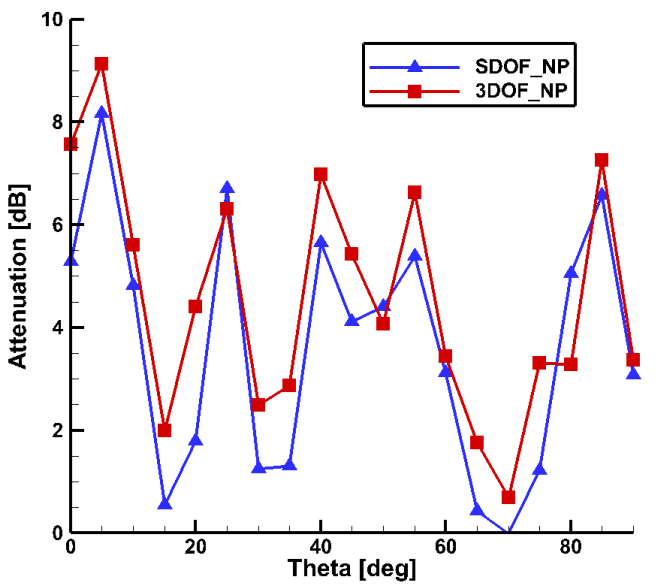

(b) Plane wave neglected in source specification.

Figure 11: Predicted mean CDL attenuation on the 10-ft. arc array for treated inlets at $f=6300 \mathrm{~Hz}$ (90\% RPMc). 